\title{
The 14-3-3 (YWHA) Proteins in Mammalian Reproduction
}

\author{
Santanu De
}

Department of Biological Sciences, Halmos College of Arts and Sciences, Nova Southeastern University, 3301 College Avenue, Fort Lauderdale, Florida 33314, United States of America

Corresponding author email: sde@nova.edu

Received: 09 June 2020 / Revised: 20 August 2020 / Accepted: 23 August 2020 / Published: 04 September 2020

\section{ABSTRACT}

The 14-3-3 (YWHA or Tyrosine 3-Monooxygenase/Tryptophan 5-Monooxygenase Activation protein) is an acidic and homologous protein family involved in regulation of key biological events including cell cycle, signal transduction and development. They are highly conserved and are expressed ubiquitously across a wide variety of species ranging from plants to animals. Seven isoforms of 14-3-3 exist in mammals, which are encoded by separate genes, have tissue-specific, developmental stage-specific as well as isoform-specific presence and consequences. It is known that these proteins play significant roles in mitosis of mammalian cells and meiosis of amphibians. However, their effects on development and functioning of mammalian gonads and germ cells are not entirely delineated. This paper, for the first time, provides a comprehensive review of the comparative expression, localization/distribution, binding interactions, and importance of the 14-3-3 proteins in gametogenesis and reproduction among multiple female and male mammalian species. Thorough understanding of these proteins in oogenesis and spermatogenesis would help elucidate the physiological basis of fertility in mammals, including humans.

Keywords: 14-3-3 proteins, 14-3-3 isoforms, YWHA, mammalian reproduction, gametogenesis.

\section{Introduction}

\subsection{Discovery, Nomenclature, Structure, and Properties of 14-3-3}

The 14-3-3 (YWHA or Tyrosine 3Monooxygenase/Tryptophan 5-Monooxygenase Activation proteins) are a family of acidic and conserved proteins expressed in numerous cells and tissues of most eukaryotic organisms, including mammals [1]. Originally, a systematic categorization of proteins in the brain detected them. The term '14-3-3' denoted the elution fraction containing these proteins following DEAE-cellulose chromatography and the position of their migration upon starch gel electrophoresis. These proteins govern critical cellular and physiological processes like transcription, signal transduction, metabolism, stress responses, malignant transformation, protein trafficking, cell cycle control, and embryonic development. Biochemical and proteomic techniques have identified as many as more than 200 proteins which can possibly interact with 14-3-3 [2], indicating 14-3-3 as a general biochemical regulator. These regulatory proteins can modify the activity(ies) of interactor(s), alter the connections of the binding partners with other factors, stabilize phosphorylation of proteins, and/or change the distribution of the binding partners in cells. They form homo- or hetero-dimers, and the monomeric molecular mass is about $30 \mathrm{kDa}$ [3].

\subsection{Isoforms of 14-3-3}

Separate genes encode the homologous proteins within the 14-3-3 family. Varied number and type(s) of 14-3-3 isoforms have been detected in different species, for example, bull, hamster, Xenopus, horseshoe crab, monkey, rat, turkey, Drosophila, and mouse [4-7]. In mammals, seven 14-3-3 isoforms have been discovered which are named beta, gamma, 
epsilon, eta, zeta, tau/theta and sigma based on their elution positions upon performing high performance liquid chromatography (HPLC) [8]. Phosphorylated beta and gamma isoforms were noted initially as alpha and delta, respectively [9]. A great degree of conservation exists among 143-3 isoforms from yeast to mammals, suggesting conservation in functional role(s) of the proteins as well. Individual isoforms of 14-3-3 can interact with the same ligand and therefore, may be interchangeable. Nevertheless, while more than one 14-3-3 isoform often associate with the same partner, varied types of homodimers or even heterodimers of 14-3-3 may differ in the way they regulate or sequester other proteins [10].

\subsection{Binding Motifs and Interactions of 14-3-3}

The 14-3-3s interact with a plethora of binding partners to coordinate a diverse range of cellular processes including metabolism, cytoskeleton rearrangements, apoptosis, cell cycle progression, protein trafficking, and transcriptional control of gene expression [11]. Dimerization of 14-3-3 proteins is significant, and their regulatory functions are impaired by point mutations disrupting dimers of 14-3-3 [12]. The role of these proteins in meiosis within mammals is not yet well defined, though they are known to be important for meiosis in amphibians and mitosis in mammalian cells. Through mechanisms involving phosphorylation, the 143-3 proteins can bind to multiple signaling molecules. Proteins 14-3-3 supplement or complement cellular processes centering around protein-protein interaction or phosphorylationdependent switching [13]. Among 14-3-3binding proteins are ones possessing phosphorthreonine and phospho-serine amino acid residues with RSXpSXP or X(Y/F)XpSXP motifs [14]. Majority of 14-3-3-interactors exist in phosphorylated state; nonetheless, phosphorylation-dependent sites with marked difference compared to these motifs occur, and certain 14-3-3-interactions do not depend on phosphorylation [10]. Binding of 14-3-3 can have multifaceted cellular effects, which would depend on the ligand's nature. Changes in the patterns of interaction may result from altered phosphorylation status of the 14-3-3-interacting motif on the binding protein. For example, 143-3-binding may be released by dephosphorylation of serine residue in a target protein, while phosphorylation of serine could foster the interaction.

\section{Proteins 14-3-3 in Mammalian Reproduction - Female}

\subsection{The 14-3-3 in Ovaries}

During each stage of ovarian folliculogenesis in the mouse, several of the mammalian 14-3-3 isoforms are expressed with isoform-specific differences and similarities in relative distribution as well as intensities [5, 1518]. Cells lining the antrum of Graafian follicles and granulosa cells of atretic follicles seem to contain all 14-3-3 isoforms to a greater extent. Certain isoforms of 14-3-3 within ovarian cells vary in their distribution among the stages of ovarian follicular development; for instance, some isoforms, but not others, are expressed in peripheral mural granulosa cells [5].

Proteins 14-3-3 can be utilized as novel markers for gynecological malignancies' screening and early diagnosis, and can be targeted specifically to treat cancers [19]. The 14-3-3 sigma/stratifin isoform is inactivated by aberration in DNA methylation, which may influence the pathogenesis of epithelial ovarian cancer in humans [20]. A potential prognostic biomarker for ovarian cancer is overexpression of 14-3-3 zeta, inhibition of which could likely be harnessed as a therapeutic strategy to augment cisplatin's antitumor activity [21]. With continuing investigations on the effect(s) of 143-3 in ovarian cancer, ovarian development, and oogenesis, it will be meaningful to unravel which 14-3-3 isoform(s) is/are present and/or abundantly expressed in somatic cells as well as germ cells of the ovary.

\subsection{The 14-3-3 in Oogenesis and Oocyte Maturation}

In bovine germinal vesicle (GV)-stage oocyte and cumulus cells, differential detergent fractionation multidimensional protein identification technology (DDF-Mud PIT) has detected 811 and 1247 proteins, respectively, 
Santanu De, Int. Ann. Sci.; Vol. 10, Issue 1, pp: 52-59, 2021

with differing expression of 371 proteins between the two cell types [22]. Compared to oocytes with intact GV, higher levels of expression of proteins related to cellular transport, cell communication, and production of energy and precursor metabolites in cumulus cells were shown in cumulus cells by systems biology modeling that involved canonical genetic pathway analysis and Gene Ontology. This indicated a probable dependence of oocytes on cumulus cells for specific cell signaling mechanisms to modulate their development, growth, and maturation. Reportedly, this was the foremost comprehensive systems biology modeling of bovine oocyte- and cumulus cellproteomes, providing a foundation for physiology and cell signaling in GV oocyte development, and comparative molecular analyses of development of oocytes in other stages.

Peptidylarginine deiminase type VI (PADI6) is a prominent interactor of 14-3-3. Phosphorylation-dependent binding between PADI6 and 14-3-3 follows oocyte maturation in the mouse [23]. Interaction of 14-3-3 with protein phosphatase $2 \mathrm{~A}(\mathrm{PP} 2 \mathrm{~A})$ regulates activity and stability of cell division cycle (CDC) proteins, CDC25A and CDC25B in particular [24].
In oocytes, the arrest at prophase I is controlled by cytoplasmic cyclic Adenosine Monophosphate (cAMP) concentration [25]. The ovarian follicular environment maintains the meiosis I arrest. High levels of cAMP in the oocyte keeps protein kinase A (PKA) active. Action of the constitutively active G-proteincoupled receptor, GPR3 enhances oocyte cytoplasmic cAMP level [26]. A reduction in oocyte cAMP concentration is triggered by the preovulatory surge of luteinizing hormone, acting on ovarian granulosa cells [27]. As a result of decreased cAMP level (and thus, a decrease in PKA activity), CDC25B phosphatase is dephosphorylated and activated, causing dephosphorylation of cyclin-dependent kinase 1 (CDK1) within maturation promoting factor (MPF), removing the prophase I arrest of the oocyte. CDC25B in the phosphorylated, inactive form is attached to and sequestered by 14-3-3 in Xenopus oocyte cytoplasm, which prevents premature germinal vesicle breakdown (GVBD) and oocyte maturation [28]. Within mammalian oocytes, 14-3-3 binds to and sequesters CDC25B in the cytoplasm, inhibiting its dephosphorylation and translocation into the nucleus, thereby arresting the oocyte at meiosis prophase 1 [29]. This is schematically presented in figure 1 [15].

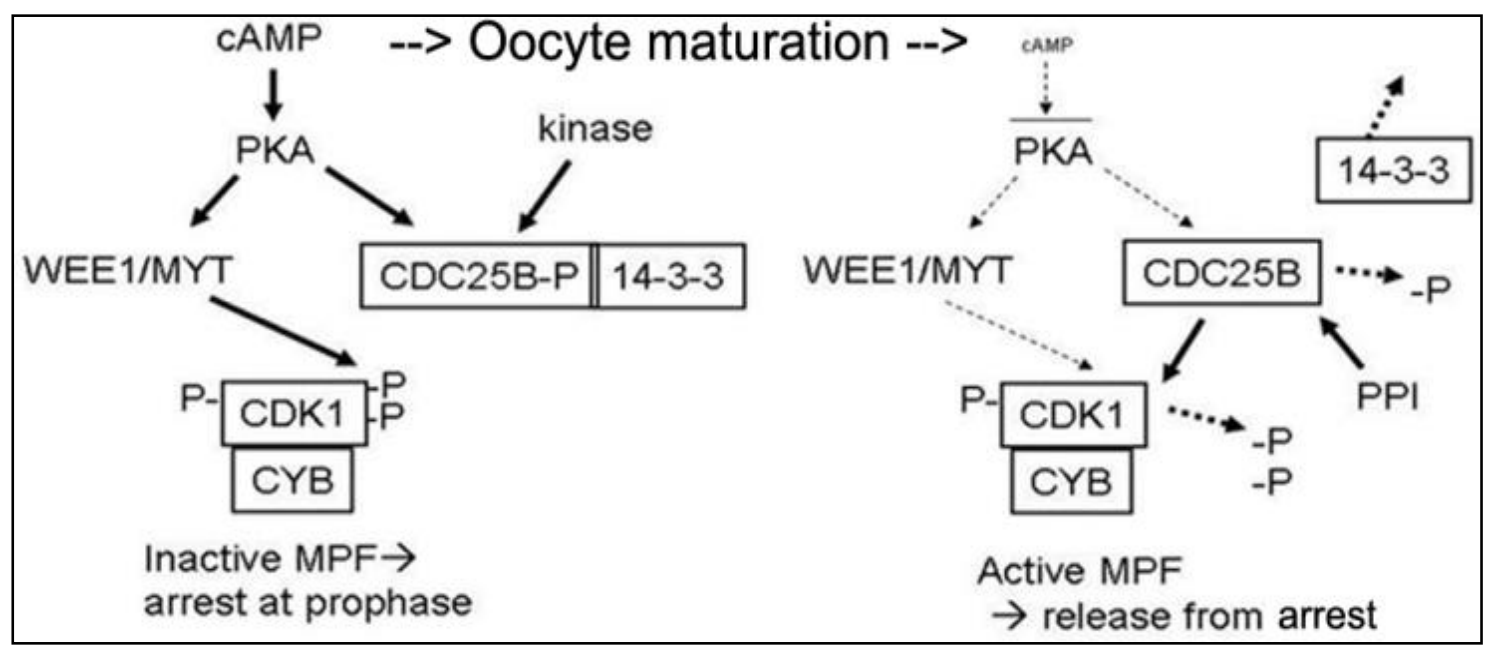

Figure 1: Schema of proteins critical to prophase I arrest of oocyte (left) and release from the arrest (right) [15].

Mitosis Promoting Factor (MPF), comprising Cyclin B (CYB) and Cyclin-Dependent Kinase 1 (CDK1), is inactive during the arrest, owing to phosphorylation of CDK1 (-P). CDC25B phosphatase is phosphorylated (by PKA or possibly some other kinase that depends on PKA), thereby binding to 14-3-3 and becoming inactivated. Pre-ovulatory surge in luteinizing hormone or removal of the oocyte from ovary decreases intracellular cAMP concentration, dephosphorylating CDC25B (potentially by protein phosphatase 1/PP1), thus being detached from 14-3-3. The activated CDC25B dephosphorylates CDK1. Thus, MPF is activated and the meiotic arrest of the oocyte is released. Studies of frog oocyte maturation, in part, form the basis of this model. 


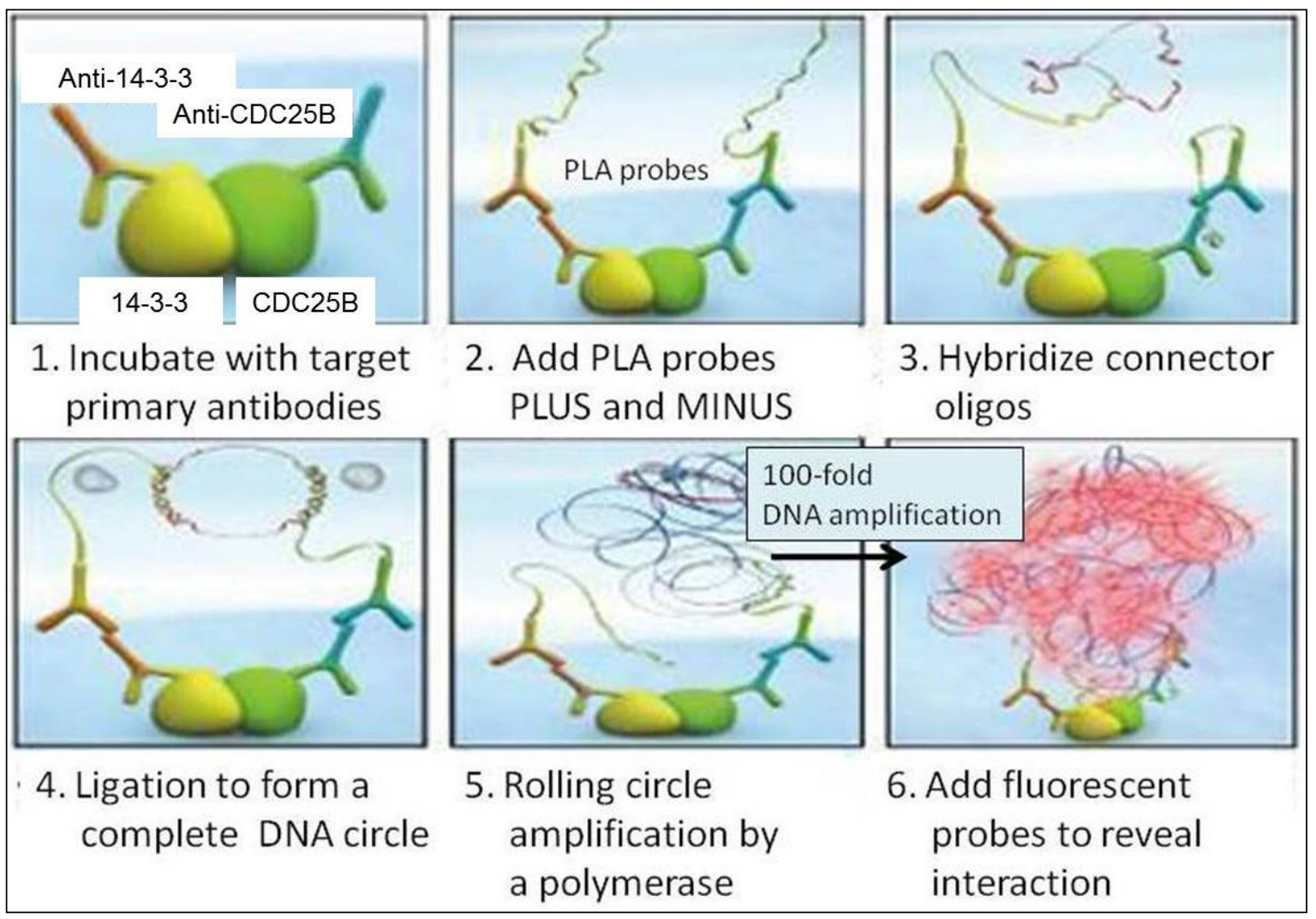

Figure 2: Schema of Duolink in situ Proximity Ligation Assay (PLA) to identify binding between two proteins [15]. The method, presented here, can detect the sites where 14-3-3 proteins interact with phosphatase CDC25B, utilizing primary antibodies generated in two individual species. Secondary antibodies were conjugated with unique oligonucleotide chains (PLA probes), targeting the primary antibodies. Enzymatic ligation joins the DNA strands if the PLA probes are within the critical distance ( $<40 \mathrm{~nm})$. By rolling circle amplification, the circular DNA molecule is amplified. A fluorescent probe is applied to hybridize with the amplified DNA, revealing the protein-protein interaction in situ. (Courtesy: Olink Bioscience).

CDC25B, among the three isoforms of CDC25 (A, B, and $\mathrm{C}$ ), is targeted for phosphorylation by PKA in fertilized eggs of the mouse [30]. Infertility was noted in female mice deficient in CDC25B isoform, apparently without any other defects [31]. Phosphorylation of CDC25B by PKA permits interaction at Ser321 of CDC25B with 14-3-3 [29]. According to other researchers, 14-3-3 epsilon binding with Ser321 of CDC25B is needed to control resumption of meiosis in mouse oocytes [32]. Application of Duolink in situ Proximity Ligation Assay (figure 2, [15]); figure courtesy: Olink Bioscience] identified interactions of all 14-3-3 isoforms with CDC25B to varying extents in cytoplasm and nucleus of mouse oocytes as well as eggs [6, 33-37].

Intracytoplasmic microinjection of mouse oocytes with the synthetic 14-3-3- blocking peptide R18 or with translationblocking 14-3-3 isoform-specific morpholino oligonucleotides (figure 3; [15]) suggested that all 14-3-3 isoforms interact with CDC25B in those cells, compared to which the binding is reduced in fertilizable eggs for all isoforms [6, 33-37].

All 14-3-3 isoforms are expressed in mouse oocytes and eggs, characterized by isoform-specific differences as well as similarities in relative distribution and amounts of expression [5]. In mouse eggs, 14-3-3 eta accumulates at the meiotic spindle region, where it was observed to colocalize and interact with alpha-tubulin [38-40]. More recent works, however, demonstrated that while message for multiple isoforms of 14-3-3 are transcribed in mouse oocytes as well as eggs, 143-3 eta and 14-3-3 epsilon are not necessary for mouse oocyte maturation, fertilization and early embryonic development [6]. 


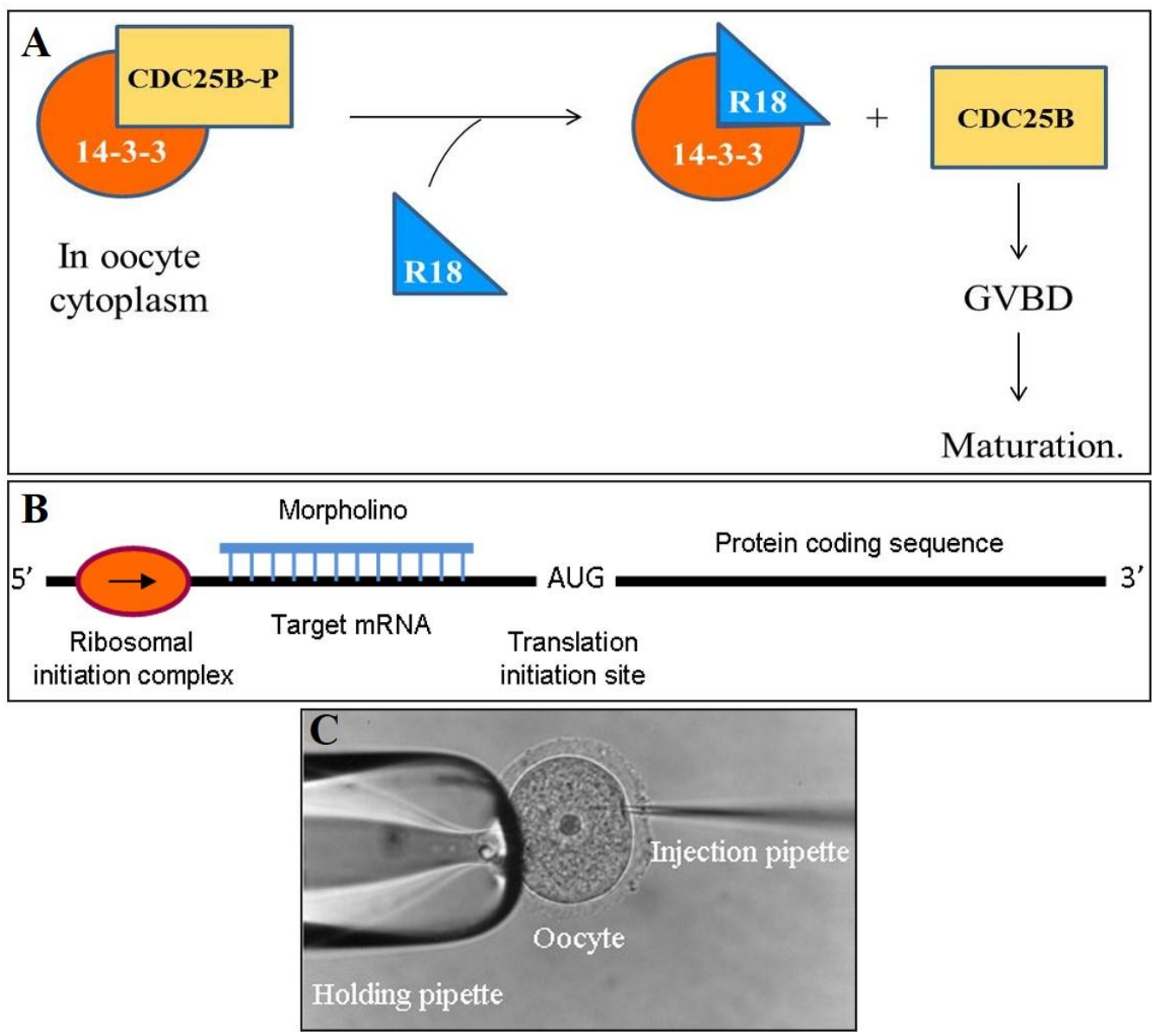

Figure 3: Schema of competitive blocking of CDC25B interaction with all 14-3-3 isoforms with synthetic peptide R18 (A), or with protein synthesis inhibitors like 14-3-3 isoform-specific morpholino oligos to stop ribosomal initiation complex from progressing to the translation start site $(\boldsymbol{B})$, achieved by a set-up for their microinjection into mouse oocyte cytoplasm $(\boldsymbol{C})$. Dissociation of CDC25B from 14-3-3 (in A) would reduce stability of its phosphorylated state, allowing oocyte maturation by germinal vesicle breakdown (GVBD) [15].

\section{Proteins 14-3-3 in Mammalian Reproduction - Male}

\subsection{The 14-3-3 in Testes}

In the testis, mitotic stem cells (spermatogonia) enter meiosis and lead to the formation of mature spermatozoa. The 14-3-3 proteins are thought to be crucial regulators within the testis. Testicular germ cells and Sertoli cells have been shown to express the 14-3-3 theta isoform [41]. Apoptosis of rat testicular germ cells, induced by experimental varicocele, is controlled by 14-3-3 epsilon which regulates Bax and $\mathrm{Bcl}-2$ expression in vitro [42]. An endogenous complex of 14-3-3 with B-raf (rapidly accelerated fibrosarcoma; a kinase) and Rap1 (Ras-proximate-1 or Ras-related protein 1; a small GTPase) was identified in mouse testis whereby it was thought to have an important regulatory role in spermiogenesis [43]. Spermiogenesis is impaired in transgenic mice expressing dominant negative Rap1 mutant [44]. The place of 14-3-3 in the pathway, however, remains to be uncovered.

Despite several proteomic studies on 143-3-binding proteins in somatic cells, the 14-3-3interactors in mouse testis were not known until recently. Using Tandem Affinity Purification of 14-3-3 zeta-bound protein complexes, mouse testis has been shown to contain as many as 135 binding partners of 14-3-3, including proteins involved in cell signaling, transcription, translation, splicing, metabolism and protein trafficking [45]. Eight of 71 novel 14-3-3-binding proteins were observed to be predominantly expressed in testis, viz. serine/threonine protein 
phosphatase type 1 isoform gamma 2 (PP1 gamma 2 or PPP1CC2), spermatogenesis associated 18 (SPATA18), phosphoglycerate kinase-2 (PGK2), testis specific gene A-2 (TSGA-2), dead box polypeptide 4 (DDX4), piwi homolog 1, protein kinase NYD-SP25 and EAN57. Some of these proteins are indispensable for spermatogenesis, which indicates that their role in germ cell division and maturation may be dependent on their association with 14-3-3.

\subsection{The 14-3-3 in Spermatogenesis and Sperm Function}

Protein 14-3-3 zeta binds to phosphorylated PP1 gamma 2 in bovine caudal epididymal spermatozoa and both appear to colocalize at the post-acrosomal region of head and principal piece [7]. This study also noted presence of 14-3-3 in spermatozoa of multifarious other species such as bull, hamster, horseshoe crab, monkey, rat, turkey, and Xenopus. Proteomic analysis of bovine sperm 14-3-3 interactome identified proteins critical in signaling and metabolism and suggested that in addition to potential regulation of a number of important sperm functions, 14-3-3 may act as an adaptor molecule for a subset of PP1 gamma 2 [46].

The multitude of 14-3-3-binding partners can be proteins influencing intracellular trafficking, cell-junction dynamics, and/or cell polarity, implicating the involvement of 14-3-3s and their interactors in germ cell movement and cell polarity [47]. For instance, during spermiogenesis and spermiation, Par6/Par3based polarity complex potentially coordinates the processes of apical ectoplasmic specialization (ES) and blood testis barrier (BTB) restructuring which take place simultaneously at the opposing ends of adjacent Sertoli cells in the seminiferous epithelium [48].

Expression of 14-3-3 beta is required for normal spermatogenesis by binding with vimentin in Sertoli cells, while its expression in malignant transformed cells in intratubular germ cell neoplasia (IGCN) and classical seminoma may result in tumorigenesis and survival of cells [49]. The 14-3-3 proteins were found to be expressed in cilia and sperm of diverse species, indicating conserved function in the motility machinery of various cells [50]. Interestingly, sperm from 14-3-3 epsilon knock-out mice exhibit reduced phosphorylation of the signal enzymes required for male fertility, PP1 gamma 2 and glycogen synthase kinase 3 (GSK3), indicating that 14-3-3 epsilon may be essential for signaling pathways responsible for sperm motility, spermatogenesis, and fertility [51, 52].

\section{Conclusions}

This paper encapsulates key research findings revealing the expression, distribution, interactions, and functional roles of the conserved, homologous, abundant, and ubiquitous family of 14-3-3 proteins underlying gametogenesis and reproductive development, compared among a gamut of mammalian species. Complex regulatory associations of these proteins with several binding partners exist, often in a phosphorylation-dependent manner, through 14-3-3 isoform-specific interactions and possible homo- or heterodimerization among the isoforms. The interchangeable and overlapping functions of 14-3-3 isoforms could be a limit our ability to determine the unique action(s) of each of them, and is recommended as a foundation for further research to provide deeper insights into the processes that govern normal fertility in mammals, including humans. The study will assist a better understanding of the molecular bases of male and female infertility and could potentially contribute to future development of novel contraceptives.

\section{Declarations}

\subsection{Acknowledgements}

The author would like to thank Dr. Douglas Kline, Dr. Srinivasan Vijayaraghavan, and Dr. Jennifer Marcinkiewicz, Kent State University, Ohio, USA for relevant help and guidance.

\subsection{Competing Interests}

The author declares no conflict of interest in preparing or publishing this article. 


\section{How to Cite this Article:}

S. De, "The 14-3-3 (YWHA) Proteins in Mammalian Reproduction”, Int. Ann. Sci., vol. 10, no. 1, pp. 52-59, Sep. 2020.

\section{References}

[1] M. B. Yaffe, "How do 14-3-3 proteins work?-Gatekeeper phosphorylation and the molecular anvil hypothesis," FEBS Lett, vol. 513, no. 1, pp. 53-7, Feb 20 2002.

[2] S. E. Meek, W. S. Lane, and H. Piwnica-Worms, "Comprehensive proteomic analysis of interphase and mitotic 14-3-3-binding proteins," J Biol Chem, vol. 279, no. 31, pp. 32046-54, Jul 302004.

[3] X. Yang et al., "Structural basis for protein-protein interactions in the 14-3-3 protein family," Proc Natl Acad Sci U S A, vol. 103, no. 46, pp. 17237-42, Nov 14 2006.

[4] S. De, "The 14-3-3 (YWHA) Proteins in Signalling and Development of the Fruit Fly, Drosophila melanogaster," International Annals of Science, vol. 9, no. 1, pp. 80-85, 2020.

[5] S. De, J. L. Marcinkiewicz, S. Vijayaraghavan, and D. Kline, "Expression of 14-3-3 protein isoforms in mouse oocytes, eggs and ovarian follicular development," BMC Res Notes, vol. 5, p. 57, Jan 232012.

[6] A. A. Eisa et al., "YWHA (14-3-3) protein isoforms and their interactions with $\mathrm{CDC} 25 \mathrm{~B}$ phosphatase in mouse oogenesis and oocyte maturation," BMC Developmental Biology, vol. 19, no. 1, p. 20, 2019/10/22 2019.

[7] Z. Huang, K. Myers, B. Khatra, and S. Vijayaraghavan, "Protein 14-3-3zeta binds to protein phosphatase PP1gamma2 in bovine epididymal spermatozoa," Biol Reprod, vol. 71, no. 1, pp. 177-84, Jul 2004.

[8] T. Ichimura et al., "Molecular cloning of cDNA coding for brain-specific 14-3-3 protein, a protein kinasedependent activator of tyrosine and tryptophan hydroxylases," Proc Natl Acad Sci U S A, vol. 85, no. 19, pp. 7084-8, Oct 1988.

[9] A. Aitken, S. Howell, D. Jones, J. Madrazo, and Y. Patel, "14-3-3 alpha and delta are the phosphorylated forms of raf-activating 14-3-3 beta and zeta. In vivo stoichiometric phosphorylation in brain at a Ser-Pro-GluLys MOTIF," J Biol Chem, vol. 270, no. 11, pp. 5706-9, Mar 171995.

[10] A. Aitken, "Functional specificity in 14-3-3 isoform interactions through dimer formation and phosphorylation. Chromosome location of mammalian isoforms and variants," Plant Mol Biol, vol. 50, no. 6, pp. 993-1010, Dec 2002

[11] A. K. Gardino, S. J. Smerdon, and M. B. Yaffe, "Structural determinants of 14-3-3 binding specificities and regulation of subcellular localization of 14-3-3ligand complexes: a comparison of the X-ray crystal structures of all human 14-3-3 isoforms," Semin Cancer Biol, vol. 16, no. 3, pp. 173-82, Jun 2006.

[12] C. M. Cahill et al., "Phosphatidylinositol 3-kinase signaling inhibits DAF-16 DNA binding and function via 14-3-3-dependent and 14-3-3-independent pathways," $J$ Biol Chem, vol. 276, no. 16, pp. 13402-10, Apr 202001.

[13] C. Mackintosh, "Dynamic interactions between 14-3-3 proteins and phosphoproteins regulate diverse cellular processes," Biochem J, vol. 381, no. Pt 2, pp. 329-42, Jul 152004

[14] K. Rittinger et al., "Structural analysis of 14-3-3 phosphopeptide complexes identifies a dual role for the nuclear export signal of 14-3-3 in ligand binding," Mol Cell, vol. 4, no. 2, pp. 153-66, Aug 1999.

[15] S. De, "Protein 14-3-3 (YWHA) isoforms and their roles in regulating mouse oocyte maturation," Kent State University, 2014.

[16] S. De, J. Marcinkiewicz, and D. Kline, "Expression of 14-3-3 Protein Isoforms in Different Stages of Follicular Development in Adult Mouse Ovaries," Biology of Reproduction, vol. 85, no. Suppl_1, p. 639, 2011.

[17] S. De and D. Kline, "The importance of 14-3-3 (YWHA) proteins in mammalian reproduction and fertility," Conference Presentation, 29th Annual Graduate Research Symposium, Kent State University 2014.

[18] S. De, B. F. Villarreal, S. Vijayaraghavan, and D. Kline, "Identification of 14-3-3 Protein Isoforms and their Differential Subcellular Distribution in Mouse Oocytes and Eggs," Mol Biol Cell \#381 vol. 22, 2011.

[19] W. Zhang et al., "The role of 14-3-3 proteins in gynecological tumors," Front Biosci (Landmark Ed), vol. 20, pp. 934-45, Jun 12015.

[20] J. Akahira et al., "Decreased expression of 14-3-3 sigma is associated with advanced disease in human epithelial ovarian cancer: its correlation with aberrant DNA methylation," Clin Cancer Res, vol. 10, no. 8, pp. 268793, Apr 152004

[21] H. J. Kim et al., "14-3-3zeta Overexpression is Associated with Poor Prognosis in Ovarian Cancer," Yonsei Med J, vol. 59, no. 1, pp. 51-56, Jan 2018.

[22] D. Peddinti, E. Memili, and S. C. Burgess, "Proteomicsbased systems biology modeling of bovine germinal vesicle stage oocyte and cumulus cell interaction," PLoS One, vol. 5, no. 6, p. e11240, Jun 212010.

[23] A. J. Snow, P. Puri, A. Acker-Palmer, T. Bouwmeester, S. Vijayaraghavan, and D. Kline, "Phosphorylationdependent interaction of tyrosine 3monooxygenase/tryptophan 5-monooxygenase activation protein (YWHA) with PADI6 following oocyte maturation in mice," Biol Reprod, vol. 79, no. 2, pp. 337-47, Aug 2008.

[24] Y. Kohama, M. Saito, M. Yada, and H. Sakurai, "Regulation of the stability and activity of CDC25A and CDC25B by protein phosphatase PP2A and 14-3-3 binding," Cell Signal, vol. 54, pp. 10-16, Feb 2019.

[25] M. Conti et al., "Role of cyclic nucleotide signaling in oocyte maturation," vol. 187, no. 1-2, pp. 153-159, 2002.

[26] L. M. Mehlmann, T. L. Jones, and L. A. Jaffe, "Meiotic arrest in the mouse follicle maintained by a Gs protein in the oocyte," Science, vol. 297, no. 5585, pp. 1343-5, Aug 232002 .

[27] L. M. Mehlmann, "Stops and starts in mammalian oocytes: recent advances in understanding the regulation of meiotic arrest and oocyte maturation," Reproduction, vol. 130, no. 6, pp. 791-9, Dec 2005.

[28] B. C. Duckworth, J. S. Weaver, and J. V. Ruderman, "G2 arrest in Xenopus oocytes depends on phosphorylation of cdc25 by protein kinase A," Proc Natl Acad Sci U S A, vol. 99, no. 26, pp. 16794-9, Dec 242002.

[29] G. Pirino, M. P. Wescott, and P. J. Donovan, "Protein kinase A regulates resumption of meiosis by phosphorylation of Cdc25B in mammalian oocytes," Cell Cycle, vol. 8, no. 4, pp. 665-70, Feb 152009. 
[30] C. Cui et al., "CDC25B acts as a potential target of PRKACA in fertilized mouse eggs," Biol Reprod, vol. 79, no. 5, pp. 991-8, Nov 2008.

[31] A. J. Lincoln et al., "Cdc25b phosphatase is required for resumption of meiosis during oocyte maturation," Nat Genet, vol. 30, no. 4, pp. 446-9, Apr 2002.

[32] J. Meng et al., "The Role of 14-3-3e Interaction with Phosphorylated Cdc25B at Its Ser321 in the Release of the Mouse Oocyte from Prophase I Arrest," PLoS One, vol. 8, no. 1, p. e53633, 2013.

[33] S. De and D. Kline, "Interactions of 14-3-3 proteins with CDC25B phosphatase in ovaries and oocytes of adult mice," Conference Presentation, 26th Annual Graduate Research Symposium, Kent State University 2011.

[34] S. De, A. Reese, and D. Kline, "Interactions of 14-3-3 (YWHA) protein isoforms with CDC25B phosphatase in mouse oocytes," Mol Biol Cell, \#1571 vol. 23, 2012.

[35] S. De, A. Reese, and D. Kline, "Interactions of 14-3-3 protein isoforms with $\mathrm{CDC} 25 \mathrm{~B}$ phosphatase in mouse oocyte maturation," Conference Presentation, Duolink User Meeting 2011.

[36] S. De, A. Reese, and D. Kline, "Duolink in situ proximity ligation assays reveal interactions of 14-3-3 protein isoforms with CDC25B phosphatase in mouse oocyte maturation," Conference Presentation, 27th Annual Graduate Research Symposium, Kent State University 2012.

[37] A. C. Detwiler, S. De, and D. Kline, "Interactions of YWHA (14-3-3) protein isoforms with CDC25B phosphatase in regulating mouse oocyte maturation," Conference Presentation, 48th Annual Meeting of the Society for the Study of Reproduction 2015.

[38] S. De and D. Kline, "Evidence for the requirement of 143-3eta (YWHAH) in meiotic spindle assembly during mouse oocyte maturation," (in eng), BMC Dev Biol, vol. 13, p. 10, Apr 12013.

[39] S. De and D. Kline, "Erratum to: evidence for the requirement of 14-3-3eta (YWHAH) in meiotic spindle assembly during mouse oocyte maturation," $B M C$ developmental biology, vol. 14, no. 1, p. 20, 2014.

[40] S. De, S. Davis, D. Letwin, C. Mozena, and D. Kline, "Protein 14-3-3 eta (YWHAH) is essential for normal meiotic spindle assembly during in vitro maturation of mouse oocytes," Mol Biol Cell, \#1967 vol. 23, 2012.

[41] J. Chaudhary and M. Skinner, "Characterization of a Novel Transcript of 14-3-3 Theta in Sertoli Cells," Journal of andrology, vol. 21, pp. 730-8, 09/10 2000.

[42] Q. Q. He, X. Wu, X. P. Liu, X. J. Yang, Z. M. Yuan, and Y. Zhang, "14-3-3 epsilon plays an important role in testicular germ cell apoptosis: A functional proteomic study of experimental varicocele," Andrologia, vol. 51, no. 6, p. e13275, 2019

[43] G. Berruti, "A novel rap1/B-Raf/14-3-3 theta protein complex is formed in vivo during the morphogenetic differentiation of postmeiotic male germ cells," Exp Cell Res, vol. 257, no. 1, pp. 172-9, May 252000.

[44] E. Aivatiadou, E. Mattei, M. Ceriani, L. Tilia, and G. Berruti, "Impaired fertility and spermiogenetic disorders with loss of cell adhesion in male mice expressing an interfering Rap1 mutant," Mol Biol Cell, vol. 18, no. 4, pp. 1530-42, Apr 2007.

[45] P. Puri, A. Acker-Palmer, R. Stahler, Y. Chen, D. Kline, and S. Vijayaraghavan, "Identification of testis 14-3-3 binding proteins by tandem affinity purification," Spermatogenesis, vol. 1, no. 4, pp. 354-365, Oct 2011.
[46] P. Puri, K. Myers, D. Kline, and S. Vijayaraghavan, "Proteomic analysis of bovine sperm YWHA binding partners identify proteins involved in signaling and metabolism," Biol Reprod, vol. 79, no. 6, pp. 1183-91, Dec 2008.

[47] S. Sun, E. W. Wong, M. W. Li, W. M. Lee, and C. Y. Cheng, "14-3-3 and its binding partners are regulators of protein-protein interactions during spermatogenesis," $J$ Endocrinol, vol. 202, no. 3, pp. 327-36, Sep 2009.

[48] E. W. Wong, D. D. Mruk, W. M. Lee, and C. Y. Cheng, "Par3/Par6 polarity complex coordinates apical ectoplasmic specialization and blood-testis barrier restructuring during spermatogenesis," Proc Natl Acad Sci U S A, vol. 105, no. 28, pp. 9657-62, Jul 152008.

[49] M. Graf, A. Brobeil, K. Sturm, K. Steger, and M. Wimmer, "14-3-3 beta in the healthy and diseased male reproductive system," Hum Reprod, vol. 26, no. 1, pp. 59-66, Jan 2011.

[50] S. Fan et al., "Polarity proteins control ciliogenesis via kinesin motor interactions," Curr Biol, vol. 14, no. 16, pp. 1451-61, Aug 242004.

[51] A. A. Eisa, "Role of 14-3-3 eta and epsilon in gametogenesis," Kent State Unversity, 2019.

[52] A. Eisa et al., "The protein YWHAE (14-3-3 epsilon) in spermatozoa is essential for male fertility," Andrology, Jul 132020 .

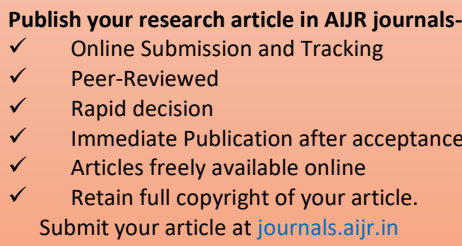

